01

\title{
Вычисление и анализ коэффициентов самоуширения линий водяного пара для спектрального диапазона $0.7-14000.0 \mathrm{~cm}^{-1}$
}

\author{
(С) В.И. Стариков \\ Томский университет систем управления и радиоэлектроники, \\ 634050 Томск, Россия \\ e-mail: vstarikov@yandex.ru
}

Поступила в редакцию 27.10.2020 г.

В окончательной редакции 27.10.2020 г.

Принята к публикации 26.11.2020 г.

В рамках полуклассического метода вычислены более 30000 коэффициентов самоуширения линий поглощения водяного пара $\gamma$ для переходов с вращательными квантовыми числами $J \leq 41, K_{a} \leq 25$ двенадцати колебательных полос из спектрального диапазона $0.7-14000.0 \mathrm{~cm}^{-1}$. Для анализа вычисленных и известных экспериментальных коэффициентов $\gamma$ применена аналитическая модель $\gamma$ (sur), зависящая от подгоночных параметров. Использованы две процедуры определения параметров $\gamma$ (sur). В первой из них параметры $\gamma$ (sur) определены для отдельных вращательных ветвей с фиксированным значением квантового числа $K_{a}=0, \ldots, 10$. Во второй процедуре параметры $\gamma($ sur) определены из подгонки $\gamma$ (sur) к 34635 экспериментальным и вычисленным значениям $\gamma$. Определена температурная зависимость $\gamma$ (sur). Полученные параметры могут быть использованы для расчета коэффициентов самоуширения линий поглощения с $J \leq 41, K_{a} \leq 13$ произвольных колебательных полос $\mathrm{H}_{2} \mathrm{O}$ из указанного диапазона.

Ключевые слова: водяной пар, самоуширение, полуклассический метод.

DOI: $10.21883 /$ OS.2021.03.50651.267-20

\section{Введение}

Изучение параметров спектральных линий поглощения водяного пара, уширенных давлением посторонних газов, представляет большой интерес для исследования атмосферы Земли, атмосфер других планет, для астрофизических исследований. При практическом анализе уширения линий молекулы $\mathrm{H}_{2} \mathrm{O}$ давлением посторонних газов используют уравнение

$$
\gamma_{L}=\gamma_{\mathrm{mol}} P_{\mathrm{mol}}+\gamma_{\mathrm{self}} P_{\mathrm{H}_{2} \mathrm{O}}
$$

в котором $\gamma_{\mathrm{L}}$ - измеренный коэффициент уширения линии, $\gamma_{\text {mol }}$ и $\gamma_{\text {self }}$ - коэффициент уширения посторонним газом и коэффициент самоуширения, $P_{\text {mol }}$ и $P_{\mathrm{H}_{2} \mathrm{O}}-$ давление постороннего газа и собственное давление $\mathrm{H}_{2} \mathrm{O}$. Для определения $\gamma_{\text {mol }}$ должно быть известно второе слагаемое в (1). Даже при очень малой концентрации водяного пара, порядка $1 \%$, вкладом самоуширения линий $\mathrm{H}_{2} \mathrm{O}$ нельзя пренебречь [1]. Согласно [2], для исследования атмосферы Земли нужны коэффициенты уширения водяного пара воздухом (т.е. азотом и кислородом) для вращательных квантовых чисел $J \sim 20$, $K_{a} \sim 15$, для исследования атмосферы Венеры необходимы коэффициенты уширения давлением углекислого газа для $J \sim 35, K_{a} \sim 20$, для различных индустриальных приложений нужны коэффициенты уширения $\gamma_{\text {mol }}$ давлением $\mathrm{N}_{2}, \mathrm{O}_{2}, \mathrm{CO}_{2}, \mathrm{H}_{2} \mathrm{O}$, Ar для $J \sim 40, K_{a} \sim 30$. Для получения информации о $\gamma_{\text {mol }}$ для таких квантовых чисел необходимы прежде всего коэффициенты самоуширения $\gamma_{\text {self }}$ водяного пара. Расчету $\gamma_{\text {self }}$ для вращательных квантовых чисел $J \leq 41, K_{a} \leq 25$ и представлению коэффициентов $\gamma_{\text {self }}$ в аналитическом виде для $J \leq 40$, $K_{a} \leq 13$ посвящена настоящая работа.

Отметим, что расчеты коэффициентов $\gamma_{\text {self }}$ проводились в литературе неоднократно, обзор результатов, полученных до 2010 г., можно найти в [2-5] . Наиболее полные расчеты $\gamma_{\text {self }}$ проведены в [5]. Рассчитанные в [5] коэффициенты $\gamma_{\text {self }}$ для 440 переходов были сравнены с экспериментальными данными для 16 колебательных полос. Среднее отклонение вычисленных значений $\gamma_{\text {self }}$ от экспериментальных значений составило 8.9\%. В настоящей работе расчеты $\gamma_{\text {self }}$ проведены для линий с намного бо́льшими значениями вращательных квантовых чисел: $J \leq 41$ и $K_{a} \leq 25$. Для проведения таких расчетов использовались волновые функции, полученные с применением производящих функций для эффективного центробежного гамильтониана молекулы $\mathrm{H}_{2} \mathrm{O}$. Такие гамильтонианы обладают улучшенным свойством сходимости (по сравнению с его полиномиальным представлением) и позволяют проводить анализ экспериментальных данных по уровням энергий (или частотам переходов) с $J \leq 42$, $K_{a} \leq 42$. Расчет коэффициентов самоуширения линий $\mathrm{H}_{2} \mathrm{O}$ с высокими $J, K_{a}$ для одной полосы $v_{2}$ проведен в [6], там же описан метод получения волновых функций.

\section{Вычисление коэффициентов самоуширения}

Вычисление коэффициентов самоуширения $\gamma_{\text {self }} \equiv \gamma$ и сдвига $\delta_{\text {self }} \equiv \delta$ для колебательно-вращательных переходов $(i)=(0,0,0)\left[J_{i} K_{a i} K_{c i}\right] \rightarrow(f)=\left(v_{1}, v_{2}, v_{3}\right)\left[J_{f} K_{a f} K_{c f}\right]$ 
(здесь $J, K_{a}, K_{c}$ - вращательные квантовые числа для нижнего, $i$, и верхнего, $f$, состояний, $v_{1}, v_{2}, v_{3}-$ колебательные квантовые числа молекулы $\mathrm{H}_{2} \mathrm{O}$ ) было проведено по формуле

$$
\begin{aligned}
& \gamma_{i f}+i \delta_{i f}=\frac{n}{c} \sum_{J 2} \rho_{J 2} \int_{0}^{\infty} v F(v) d v \\
& \times \int_{0}^{\infty} b d b\left[1-\left(1-S_{2}^{\text {middle }^{\prime}}\right)\right. \\
& \left.\times \exp \left(-i S_{1}-S_{2, i}^{\text {outer }}-S_{2, f}^{\text {outer }}-S_{2}^{\text {middle }^{\prime \prime}}\right)\right]
\end{aligned}
$$

из полуклассической схемы Робера-Бонами [7]. В этой формуле $n$ - плотность газа, $c$ - скорость света, $J_{2}$ - квантовые числа для вращательного состояния возмущающей молекулы $\mathrm{H}_{2} \mathrm{O}, \rho_{J 2}-$ статистический вес этого состояния, $F(v)$ - больцмановская функция распределения молекул по скоростям $v, b-$ прицельное расстояние, $S_{1}(b)$ и $S_{2}(b)$ - функции прерывания первого и второго порядков соответственно. Они определяются потенциалом взаимодействия через формулы, приведенные в $[7,8]$. В межмолекулярном потенциале $\mathrm{H}_{2} \mathrm{O}-\mathrm{H}_{2} \mathrm{O}$ учитывались диполь-дипольное, диполь-квадрупольное, диполь-октупольное, квадрупольквадрупольное и поляризационное взаимодействия. Резонансные функции $S_{2}(b)$ были вычислены в приближении прямолинейных траекторий по формулам из [8].

Зависимость эффективного дипольного момента $\mu$ от вращательных квантовых чисел была выбрана в форме паде-аппроксиманта

$$
\begin{aligned}
& \mu(v, J, K)=\mu(v) \\
& +\frac{\left(h_{200} J(J+1)+h_{020} K^{2}\right)^{2}}{h_{200} J(J+1)+h_{020} K^{2}-h_{400}[J(J+1)]^{2}-h_{220} J(J+1) K^{2}-h_{040} K^{4}} .
\end{aligned}
$$

Здесь $K \equiv K_{a}, \mu(v)$ - значения дипольного момента в данном колебательном состоянии $(v) \equiv\left(v_{1}, v_{2}, v_{3}\right)$, они брались из [9], параметры $h_{200}, \ldots, h_{040}$ приведены в $[3,4,10]$. Эти параметры зависят от квантового числа $v_{2}$, связанного с колебанием большой амплитуды в молекуле $\mathrm{H}_{2} \mathrm{O}$. Зависимость средней поляризуемости $\alpha(v)$ $\left(\right.$ в $\left.\AA^{3}\right)$ от колебательных квантовых чисел определялась [11] по формуле

$$
\alpha(v)=1.469+0.39 v_{1}+0.22 v_{2}+0.40 v_{3} .
$$

Зависимость $\alpha$ от вращательных чисел не учитывалась.

\section{Сравнение с экспериментальными данными}

Качество вычисления коэффициентов уширения $\gamma(\mathrm{cal})$ для отдельного перехода $(i) \rightarrow(f)$ контролировалось с помощью величины

$$
\chi=100 \%|1-\gamma(\mathrm{cal}) / \gamma(\exp )|,
$$

\begin{tabular}{|c|c|c|c|c|}
\hline$v_{1} v_{2} v_{3}$ & $N$ & $\operatorname{Max}\left(J_{i}, K_{a i}\right)$ & Источник & $\chi_{\mathrm{av}}, \%$ \\
\hline 000 & 100 & $(16,7)$ & {$[12]$} & 3.1 \\
\hline 010 & 711 & $(16,10)$ & {$[12]$} & 3.1 \\
\hline 001 & 74 & $(9,5)$ & [13] & 5.6 \\
\hline 110 & 64 & $(11,4)$ & {$[14]$} & 6.6 \\
\hline 0111 & 183 & $(13,7)$ & {$[14]$} & 5.3 \\
\hline 021 & 77 & $(10,5)$ & [14] & 3.9 \\
\hline 120 & 48 & $(9,4)$ & [14] & 4.2 \\
\hline 200 & 76 & $(10,6)$ & [14] & 5.2 \\
\hline 002 & 50 & $(9,7)$ & {$[14]$} & 4.4 \\
\hline 202 & 12 & $(8,3)$ & {$[15]$} & 3.8 \\
\hline 221 & 53 & $(7,5)$ & {$[15]$} & 6.4 \\
\hline 301 & 90 & $(9,6)$ & {$[15]$} & 3.9 \\
\hline
\end{tabular}

Таблица 1. Значения величины $\chi_{\mathrm{av}}$, полученные при сравнении вычисленных и экспериментальных [12-15] коэффициентов самоуширения линий водяного пара для 12 колебательных полос $(0,0,0) \rightarrow\left(v_{1}, v_{2}, v_{3}\right)$

Примечание. $N$ - число переходов в полосе, суммарное число перехо-

\begin{tabular}{|c|c|c|c|c|c|}
\hline$v_{1} v_{2} v_{3}$ & $N$ & $\operatorname{Max}\left(J_{i}, K_{a i}\right)$ & $v_{1} v_{2} v_{3}$ & $N$ & $\operatorname{Max}\left(J_{i}, K_{a i}\right)$ \\
\hline 000 & 4305 & $(41,13)$ & 110 & 4332 & $(25,10)$ \\
\hline $\begin{array}{lll}0 & 1 & 0\end{array}$ & 4601 & $(41,10)$ & $\begin{array}{lll}0 & 0 & 2\end{array}$ & 2067 & $(16,10)$ \\
\hline 030 & 3918 & $(25,10)$ & 200 & 2106 & $(16,10)$ \\
\hline 100 & 4086 & $(25,10)$ & 202 & 602 & $(11,10)$ \\
\hline 001 & 3241 & $(25,10)$ & 221 & 331 & $(11,10)$ \\
\hline 011 & 3158 & $(25,10)$ & 301 & 331 & $(11,10)$ \\
\hline
\end{tabular}
дов равно $1557, \operatorname{Max}\left(J_{i}, K_{a i}\right)$ - максимальные значения вращательных квантовых чисел $J_{i}$ и $K_{a i}$ нижнего уровня энергии, вовлеченного в переход.

Таблица 2. Переходы, для которых вычислены значения коэффициентов $\gamma(\mathrm{cal})$, используемые для анализа по аналитической модели $\gamma$ (sur)

Примечание. Суммарное число переходов равно 33078.

а для совокупности переходов (например, для всей колебательной полосы) - с помощью величины

$$
\chi_{a v}=100 \% \cdot \sum_{i=1}^{N}\left|1-\gamma_{i}(\mathrm{cal}) / \gamma_{i}(\exp )\right| / N,
$$

где $N$ - число используемых данных для $\gamma(\exp )$.

В табл. 1 показаны значения величины $\chi_{\mathrm{av}}$, полученные при сравнении вычисленных и экспериментальных коэффициентов самоуширения линий водного пара для анализируемых в работе колебательных полос.

Из табл. 1 видно, что экспериментальные данные для рассмотренных 12 полос описываются с погрешностью от 3.1 до $6.6 \%$, судя по значениям $\chi_{\text {av }}$. Этот результат сравним с тем, что получается с применением современных полуклассических методов расчета [5].

Для 12 колебательных полос, указанных в табл. 2, были вычислены 33078 значений $\gamma(\mathrm{cal})$ для вращательных квантовых чисел $J \leq 41, K_{a} \leq 25$ нижнего состояния, вовлеченного в переход. 


\section{Анализ данных}

Табличное представление вычисленных 33078 значений коэффициентов $\gamma$ является громоздким. Поэтому желательно иметь аналитическую формулу, которая позволяет в компактном виде представить полученную информацию и проводить вычисления $\gamma$ для переходов с большими вращательными и колебательными числами для конечного состояния. Для коэффициентов самоуширения $\gamma$ такая формула была предложена ранее в $[3,4,16]$ и имеет следующий вид:

$$
\begin{aligned}
& \gamma(\operatorname{sur})=x_{1}+x_{2}\left\{1 / \operatorname{Cosh}\left[x_{3}\left(K_{a i}-x_{4}\right)\right]\right. \\
& \left.+1 / \operatorname{Cosh}\left[x_{3}\left(K_{a f}-x_{4}\right)\right]\right\}
\end{aligned}
$$

В этой формуле $x_{1}, x_{2}, x_{3}, x_{4}-$ функции квантовых чисел $J_{i}, J_{f}$, зависящие от варьируемых параметров, которые определяются методом наименьших квадратов из известных значений $\gamma$. Для увеличения точности восстановления известных $\gamma$ в настоящей работе введена зависимость $\gamma($ sur $)$ от вращательных $\left(K_{c i}, K_{c f}\right)$ и колебательных $\left(v_{1}, v_{2}, v_{3}\right)$ квантовых чисел молекулы $\mathrm{H}_{2} \mathrm{O}$. В предлагаемой модели функция $x_{2}$ выглядит следующим образом:

$$
\begin{aligned}
& x_{2}=\left(x_{20}+x_{24}\left(J_{f}-K_{c f}\right)+x_{25}\left|K_{a i}-K_{a f}\right|\right)\left(1+t_{21} v_{1}\right. \\
& \left.+t_{22} v_{2}+t_{23} v_{3}\right) / \operatorname{Cosh}\left[x_{21}\left(J_{i}+J_{f}\right)\right. \\
& \left.+x_{22}\left(K_{c i}+K_{c f}\right)+x_{23}\left(J_{i}+J_{f}\right)\left(K_{c i}+K_{c f}\right)\right]
\end{aligned}
$$

а функции $x_{k}(k=1,3,4)$ имеют вид

$$
x_{k}=x_{k 0}+x_{k 1}\left(J_{i}+J_{f}\right) .
$$

В формулах (7)-(9) коэффициенты $x_{2 m}, t_{2 p}, x_{1 l}, x_{3 l}, x_{4 l}$ $(m=0-5 ; p=1-3 ; l=0,1)$ являются подгоночными параметрами. Параметры $t_{2 p}$ определяют зависимость коэффициентов $\gamma$ от колебательных квантовых чисел. Для определения параметров модели $\gamma$ (sur) по формулам (7)-(9) использовались 33078 вычисленных значений коэффициентов $\gamma$ (для 12 полос из табл. 2) и 1557 экспериментальных значений $\gamma$ (для 12 полос из табл. 1), т. е. всего 34635 значений $\gamma$ с вращательными квантовыми числами $J_{i} \leq 41, K_{a i} \leq 13$.

Две процедуры определения параметров $\gamma$ (sur) были применены в настоящей работе. В первой процедуре выражения (7)-(9) подгонялись к коэффициентам $\gamma$, измеренным и вычисленным для отдельных вращательных ветвей $(0,0,0)\left[J_{i} K_{a i} K_{c i}\right] \rightarrow\left(v_{1}, v_{2}, v_{3}\right)\left[J_{f} K_{a f} K_{c f}\right]$ с фиксированным значением вращательного квантового числа $K_{a i}=0,1,2, \ldots, 10$. Найденные в этой подгонке параметры модели $\gamma($ sur) и статистический анализ разностей между $\gamma$ (sur) и используемыми данными приведены в табл. 3.

В этой таблице величины $\chi$ и $\chi_{\mathrm{av}}$ определены формулами (5) и (6), в которых $\gamma(\mathrm{cal})$ и $\gamma(\exp )$ - вычисленные $\gamma$ (sur) по модели (7)-(9) и используемые в подгонке значения $\gamma$ соответственно. Статистический анализ разностей между $\gamma$ (sur) и используемыми данными для всех 34195 значений $\gamma$ дает следующий результат:

$$
\begin{gathered}
0 \leq \chi \leq 5 \% \quad \text { для } 13037 \text { линий }(38.2 \%), \\
5 \%<\chi \leq 10 \% \quad \text { для } 9448 \text { линий }(27.6 \%), \\
10 \%<\chi \leq 20 \% \quad \text { для } 9031 \text { линий }(26.4 \%), \\
20 \% \leq \chi \leq 50 \% \quad \text { для } 2637 \text { линий }(7.7 \%), \\
\chi>50 \% \quad \text { для } 44 \text { линий }(0.1 \%) .
\end{gathered}
$$

Согласно (10), для 22485 линий (65.8\% всех линий) $\chi \leq 10 \%$, согласно табл. $36.2<\chi_{\mathrm{av}}<11.4 \%$. Точность вычислений $\gamma$ по аналитической модели, судя по величине $\chi_{\mathrm{av}}$, уступает точности вычислений $\gamma$ в полуклассическом методе (табл. 1), но аналитическая модель позволяет легко проводить расчеты $\gamma$ для широкого диапазона вращательных и колебательных квантовых чисел без привлечения потенциала взаимодействия, волновых функций и частот переходов в молекуле $\mathrm{H}_{2} \mathrm{O}$.

Из трех параметров $t_{21}, t_{22}$ и $t_{23}$, которые описывают зависимость коэффициентов $\gamma$ от колебательных квантовых чисел, только один параметр, $t_{22}$, является хорошо определенным параметром. Два других параметра, $t_{21}$ и $t_{23}$, могут быть фиксированы на нулевых значениях без потери точности вычислений. Это значит, что коэффициенты самоуширения $\gamma$ существенно зависят от колебательного квантового числа $v_{2}$, связанного с изгибным колебанием, но слабо зависят от колебательных квантовых чисел $v_{1}$ и $v_{3}$, связанных с валентными колебаниями. Этот факт является следствием того, что главным вкладом в потенциале взаимодействия $\mathrm{H}_{2} \mathrm{O}-\mathrm{H}_{2} \mathrm{O}$ является диполь-дипольное взаимодействие, а дипольный момент молекулы $\mathrm{H}_{2} \mathrm{O}$ зависит от $v_{2}$ сильнее, чем от $v_{1}$ и $v_{3}[9]$.

Рис. 1-3 дают примеры использования модели $\gamma$ (sur) и параметров из табл. 3 для расчета асимптотического поведения $\gamma$ для трех ветвей перехода $(0,0,0)\left[J_{i} K_{a i} K_{c i}\right] \rightarrow\left(v_{1}, v_{2}, v_{3}\right)\left[J_{f} K_{a f} K_{c f}\right]$. Здесь же проведено сравнение с известными экспериментальными данными.

По второй процедуре вычисления коэффициентов самоуширения выражения (7)-(9) подгонялись одновременно к 34635 значениям коэффициентов, измеренных и вычисленных для 12 колебательных полос молекулы $\mathrm{H}_{2} \mathrm{O}$, с $J_{i} \leq 40, K_{a i} \leq 13$. Найденные параметры приведены в табл. 4.

Статистический анализ разностей между $\gamma($ sur $)$ и используемыми данными для всех 34635 значений $\gamma$ дает следующий результат:

$$
\begin{gathered}
0 \leq \chi_{\gamma} \leq 5 \% \quad \text { для } 10435 \text { линий }(30.1 \%), \\
5 \%<\chi_{\gamma} \leq 10 \% \quad \text { для } 8948 \text { линий }(25.8 \%), \\
10 \%<\chi_{\gamma} \leq 20 \% \quad \text { для } 10803 \text { линий }(32.2 \%), \\
20 \% \leq \chi_{\gamma} \leq 50 \% \quad \text { для } 4446 \text { линий }(12.8 \%),
\end{gathered}
$$


Таблица 3. Параметры модели $\gamma$ (sur), найденные в подгонке выражений (7)-(9) к вычисленным и экспериментальным $(T=296 \mathrm{~K})$ коэффициентам самоуширения линий отдельных вращательных ветвей с фиксированным значением $K_{a i}=0,1, \ldots, 10$ двенадцати колебательных полос водяного пара

\begin{tabular}{|c|c|c|c|c|}
\hline Параметр & $K_{a i}=0$ & $K_{a i}=1$ & $K_{a i}=2$ & $K_{a i}=3$ \\
\hline$x_{10}$ & $0.9709(168) \cdot 10^{-1}$ & $0.9938(154) \cdot 10^{-1}$ & $0.9224(220) \cdot 10^{-1}$ & $0.7457(195) \cdot 10^{-1}$ \\
\hline$x_{20}$ & $0.2044(15)$ & $0.2089(13)$ & $0.2234(17)$ & $0.2280(13)$ \\
\hline$x_{21}$ & $0.1061(63)$ & $0.1104(32)$ & $0.1095(22)$ & $0.9308(178) \cdot 10^{-1}$ \\
\hline$x_{22}$ & $-0.1512(72)$ & $-0.1681(38)$ & $-0.1874(29)$ & $-0.1709(25)$ \\
\hline$x_{23}$ & $-0.1443(78) \cdot 10^{-2}$ & $-0.1004(57) \cdot 10^{-2}$ & $-0.2692(513) \cdot 10^{-3}$ & 0.0 \\
\hline$x_{24}$ & 0.0 & 0.0 & 0.0 & 0.0 \\
\hline$t_{21}$ & 0.0 & 0.0 & 0.0 & 0.0 \\
\hline$t_{22}$ & $-0.2919(266) \cdot 10^{-1}$ & $-0.3109(195) \cdot 10^{-1}$ & $-0.3770(186) \cdot 10^{-1}$ & $-0.3094(189) \cdot 10^{-1}$ \\
\hline$t_{23}$ & 0.0 & 0.0 & 0.0 & 0.0 \\
\hline$x_{30}$ & $0.2334(102)$ & $0.2550(50)$ & $0.2616(33)$ & $0.2207(27)$ \\
\hline$x_{31}$ & 0.0 & 0.0 & 0.0 & 0.0 \\
\hline \multirow[t]{6}{*}{$N$} & 1363 & 3208 & 3930 & 3699 \\
\hline & $0 \leq \chi \leq 5 \%$ (732 линий $)$ & $0 \leq \chi \leq 5 \%$ (1720 линий $)$ & $0 \leq \chi \leq 5 \%$ (2025 линий $)$ & $0 \leq \chi \leq 5 \%(1629$ линий $)$ \\
\hline & $5 \% \leq \chi \leq 10 \%(422)$ & $5 \% \leq \chi \leq 10 \%(847)$ & $5 \% \leq \chi \leq 10 \%(1049)$ & $5 \% \leq \chi \leq 10 \%(1083)$ \\
\hline & $10 \% \leq \chi \leq 20 \%(165)$ & $10 \% ? \chi \leq 20 \%(509)$ & $10 \% \leq \chi \leq 20 \%(679)$ & $10 \% \leq \chi \leq 20 \%(809)$ \\
\hline & $20 \% \leq \chi \leq 50 \%(37)$ & $20 \% \leq \chi \leq 50 \%(123)$ & $20 \% \leq \chi \leq 50 \%(175)$ & $20 \leq \chi \leq 50 \%(178)$ \\
\hline & $\chi>50 \%(7)$ & $\chi>50 \%(9)$ & $\chi>50 \%(2)$ & $\chi>50 \%(0)$ \\
\hline$\chi_{\mathrm{av}}$ & $6.2 \%$ & $6.5 \%$ & $6.6 \%$ & $7.5 \%$ \\
\hline$n_{1}$ & $0.36(0.07)$ & 0.0 & $0.28(0.08)$ & 0.0 \\
\hline$n_{2}$ & $0.92(0.03)$ & $1.13(0.02)$ & $0.89(0.02)$ & $1.10(0.03)$ \\
\hline \multirow[t]{2}{*}{$n_{3}$} & 0.0 & $1.17(0.11)$ & 0.0 & $0.63(0.07)$ \\
\hline & $K_{a i}=4$ & $K_{a i}=5$ & $K_{a i}=6$ & $K_{a i}=7$ \\
\hline$x_{10}$ & $0.5077(355) \cdot 10^{-1}$ & $0.4398(539) \cdot 10^{-1}$ & $0.5293(790) \cdot 10^{-1}$ & 0.0 \\
\hline$x_{20}$ & $0.2215(18)$ & $0.2049(27)$ & $0.2068(38)$ & $0.3329(114)$ \\
\hline$x_{21}$ & $0.8196(183) \cdot 10^{-1}$ & $0.8206(191) \cdot 10^{-1}$ & $0.8128(223) \cdot 10^{-1}$ & $0.3226(289) \cdot 10^{-1}$ \\
\hline$x_{22}$ & $-0.1498(27)$ & $-0.1467(31)$ & $-0.1429(37)$ & $-0.8585(265) \cdot 10^{-1}$ \\
\hline$x_{23}$ & 0.0 & 0.0 & 0.0 & 0.0 \\
\hline$x_{24}$ & 0.0 & 0.0 & $0.2256(241) \cdot 10^{-1}$ & $0.1786(260) \cdot 10^{-1}$ \\
\hline$t_{21}$ & 0.0 & 0.0 & 0.0 & 0.0 \\
\hline$t_{22}$ & $-0.3046(225) \cdot 10^{-1}$ & $-0.2953(245) \cdot 10^{-1}$ & $-0.3011(2853) \cdot 10^{-1}$ & $-0.2290(237) \cdot 10^{-1}$ \\
\hline$t_{23}$ & 0.0 & 0.0 & 0.0 & 0.0 \\
\hline$x_{30}$ & $0.1798(26)$ & $0.1466(28)$ & $0.2579(93)$ & $0.3616(106)$ \\
\hline$x_{31}$ & 0.0 & 0.0 & 0.0 & $-0.4008(324) \cdot 10^{-2}$ \\
\hline$n_{1}$ & 0.0 & 0.0 & 0.0 & 0.0 \\
\hline$n_{2}$ & $1.22(0.03)$ & $0.65(0.03)$ & $0.61(0.04)$ & $0.44(0.02)$ \\
\hline$n_{3}$ & 0.0 & 0.0 & 0.0 & 0.0 \\
\hline \multirow[t]{6}{*}{$N$} & 4061 & 3601 & 3431 & 3160 \\
\hline & $0<\chi ? 5 \%(1408)$ & $0<\chi \leq 5 \%(983)$ & $0<\chi \leq 5 \%(979)$ & $0<\chi \leq 5 \%(830)$ \\
\hline & $5 \%<\chi \leq 10 \%(1114)$ & $5 \%<\chi \leq 10 \%(1035)$ & $5 \%<\chi \leq 10 \%(898)$ & $5 \%<\chi \leq 10 \%(788)$ \\
\hline & $10 \%<\chi \leq 20 \%(1160)$ & $10 \%<\chi \leq 20 \%(1259)$ & $10 \%<\chi \leq 10 \%(1241)$ & $10 \%<\chi \leq 10 \%(1066)$ \\
\hline & $20 \%<\chi \leq 50 \%(379)$ & $20 \%<\chi \leq 50 \%(320)$ & $20 \%<\chi \leq 50 \%(312)$ & $20 \%<\chi \leq 50 \%(465)$ \\
\hline & $\chi>50 \%(0)$ & $\chi>\overline{50} \%(4)$ & $\chi>50 \%(1)$ & $\chi>50 \%(11)$ \\
\hline \multirow[t]{2}{*}{$\chi_{\mathrm{av}}$} & $9.2 \%$ & $10.2 \%$ & $10.9 \%$ & $11.4 \%$ \\
\hline & $K_{a i}=8$ & $K_{a i}=9$ & $K_{a i}=10$ & \\
\hline$x_{10}$ & 0.0 & 0.0 & 0.0 & \\
\hline$x_{20}$ & $0.4988(148)$ & $0.4746(163)$ & $0.2874(219)$ & \\
\hline$x_{21}$ & 0.0 & 0.0 & 0.0 & \\
\hline$x_{22}$ & $0.5358(94) \cdot 10^{-1}$ & $0.4833(107) \cdot 10^{-1}$ & $0.3592(110) \cdot 10^{-1}$ & \\
\hline$x_{23}$ & 0.0 & 0.0 & 0.0 & \\
\hline$x_{24}$ & $0.3857(422) \cdot 10^{-1}$ & $0.6741(673) \cdot 10^{-1}$ & $0.9174(902) \cdot 10^{-1}$ & \\
\hline$t_{21}$ & 0.0 & 0.0 & 0.0 & \\
\hline$t_{22}$ & $-0.3064(234) \cdot 10^{-1}$ & $-0.3252(208) \cdot 10^{-1}$ & $-0.3156(199) \cdot 10^{-1}$ & \\
\hline$t_{23}$ & 0.0 & 0.0 & 0.0 & \\
\hline
\end{tabular}


Таблица 3 (продолжение)

\begin{tabular}{l|c|c|c}
\hline Параметр & $K_{a i}=8$ & $K_{a i}=9$ & $K_{a i}=10$ \\
\hline$x_{30}$ & $0.4556(84)$ & $0.4438(77)$ & $0.3927(73)$ \\
$x_{31}$ & $-0.5887(140) \cdot 10^{-2}$ & $-0.4504(131) \cdot 10^{-2}$ & $-0.2641(110) \cdot 10^{-2}$ \\
$N$ & 2839 & 2606 & 2297 \\
& $0<\chi \leq 5 \%(886)$ & $0<\chi \leq 5 \%(931)$ & $0<\chi \leq 5 \%(914)$ \\
& $5 \%<\chi \leq 10 \%(763)$ & $5 \%<\chi \leq 10 \%(730)$ & $5 \%<\chi \leq 10 \%(719)$ \\
& $10 \%<\chi \leq 10 \%(817)$ & $10 \%<\chi \leq 10 \%(759)$ & $10 \%<\chi \leq 10 \%(567)$ \\
$\chi_{\mathrm{av}}$ & $20 \%<\chi \leq 50 \%(366)$ & $20 \%<\chi \leq 50 \%(184)$ & $20 \%<\chi \leq 50 \%(96)$ \\
$n_{1}$ & $\chi>50 \%(7)$ & $\chi>50 \%(2)$ & $\chi>50 \%(1)$ \\
$n_{2}$ & $10.5 \%$ & $8.9 \%$ & $7.7 \%$ \\
$n_{3}$ & 0.0 & 0.0 & 0.0 \\
& $0.43(0.03)$ & $0.43(0.03)$ & $0.38(0.04)$ \\
\end{tabular}

Примечание. Параметры $x_{20}$ и $x_{24}$ имеют размерность $\mathrm{cm}^{-1} / \mathrm{atm}$, другие параметры безразмерны, $x_{25}$ в $(8)$ и $x_{4}$ в $(7)$ равны нулю для всех ветвей.

Таблица 4. Параметры модели $\gamma($ sur $)$, найденные подгонкой выражений (7)-(9) к 34635 вычисленным и измеренным $(T=296 \mathrm{~K})$ коэффициентам самоуширения линий водяного пара с $0 \leq J_{i} \leq 41,0 \leq K_{a i} \leq 13$ одновременно

\begin{tabular}{l|c|c|c}
\hline Параметр & Численное значение & Параметр & Численное значение \\
\hline$x_{10}$ & $0.3126(204) \cdot 10^{-1}$ & $x_{25}$ & $0.1647(176) \cdot 10^{-2}$ \\
$x_{20}$ & $0.2462(11)$ & $x_{30}$ & $0.2412(15)$ \\
$x_{21}$ & $-0.6139(67) \cdot 10^{-1}$ & $x_{31}$ & $-0.2124(28) \cdot 10^{-2}$ \\
$x_{22}$ & $0.1359(10)$ & $t_{21}$ & 0.0 \\
$x_{23}$ & $-0.2927(77) \cdot 10^{-3}$ & $t_{22}$ & $-0.2876(75) \cdot 10^{-1}$ \\
$x_{24}$ & $0.1003(163) \cdot 10^{-2}$ & $t_{23}$ & 0.0 \\
$\chi_{\mathrm{av}}$ & $10.4 \%$ & $n_{1}$ & 0.0 \\
$n_{2}$ & $0.87(0.01)$ & $n_{3}$ & $0.33(0.02)$
\end{tabular}

Примечание. Параметры $x_{20}, x_{24}, x_{25}$ определены в $\mathrm{cm}^{-1} / \mathrm{atm}$, остальные параметры безразмерны; функция $x_{4}=0$.

$$
\chi_{\gamma}>40 \% \text { для } 4 \text { линий }(0.02 \%) \text {. }
$$

В этой процедуре $\chi_{\gamma} \leq 10.0 \%$ для 19383 линий (55.9\%) и $\chi_{\mathrm{av}}=10.4 \%$. В отличие от первой процедуры параметры для $\gamma$ (sur) из табл. 4 получены для всех ветвей с $K_{a i}=0,1, \ldots, 13$ одновременно и позволяют проводить вычисления коэффициентов $\gamma$ для значений квантового числа $K_{a i} \leq 15$. Пример вычисления $\gamma$ для переходов с большими $K_{a i}$ представлен на рис. 4 .

\section{Зависимость от температуры}

В работе была рассчитана температурная зависимость модели (7)-(9). Для этой цели были вычислены значения 3000 коэффициентов $\gamma$ для температур $T=200$, 400 и $500 \mathrm{~K}$ для переходов с максимальными значениями $J_{i}=40, K_{a i}=10$ в колебательных полосах $v_{2}$, $2 v_{1}+2 v_{2}+v_{1}, 2 v_{1}+2 v_{1}, 3 v_{1}+v_{1}$. Пример асимптотического поведения коэффициентов самоуширения линий вращательной ветви $[J 0 J] \rightarrow[J+11 J+1]$ колебательной полосы $v_{2}$, вычисленных для различных температур, показан на рис. 5 .

В аналитической модели (7)-(9) была введена зависимость величин $x_{k}(k=1,2,3)$ от температуры через соотношение

$$
x_{k}(T)=x_{k}\left(T_{0}\right)\left(\frac{T_{0}}{T}\right)^{n_{k}},
$$

содержащее варьируемые параметры $n_{k}$, при $T_{0}=296 \mathrm{~K}$. Эти параметры были определены из подгонки выражений (7),(12) к вычисленным коэффициентам $\gamma(T)$, при этом $x_{k}\left(T_{0}\right)$ были фиксированы на значениях из табл. 3, 4. Значения ненулевых параметров $n_{k}$ приведены в табл. 3,4 .

\section{Обсуждение}

Предложенную аналитическую модель (7)-(9) очень просто использовать для расчетов коэффициентов самоуширения линий поглощения молекулы водяного пара из широкого спектрального интервала $0.7-14000.0 \mathrm{~cm}^{-1}$, поскольку процедура расчета не требует привлечения информации о межмолекулярном потенциале взаимодействия, волновых функциях и частотах переходов в молекуле. Средняя точность $\chi_{\mathrm{av}}$ (5) восстановления экспериментальных и вычисленных в полуклассическом методе данных согласно табл. 3 меняется от 6.2 до 10.5\%. В табл. 5 сравниваются вычисленные по модели (7)-(9) 


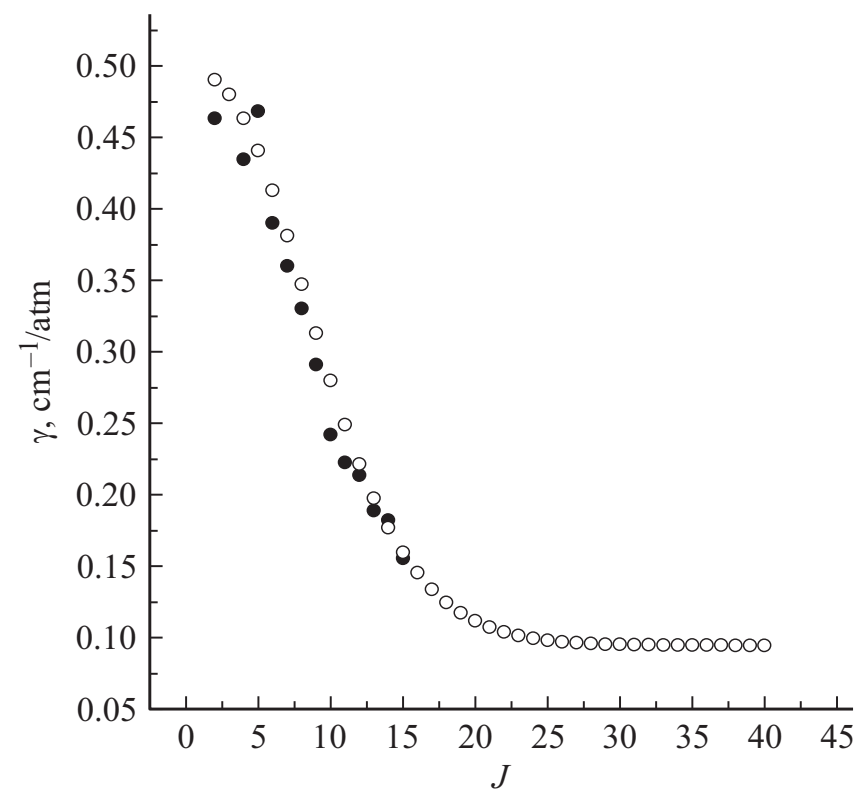

Рис. 1. Экспериментальные [12] (темные символы) и вычисленные (светлые символы) коэффициенты самоуширения линий $[J 0 J] \rightarrow[J-11 J-1]$ полосы $v_{2}$ молекулы водяного пара.

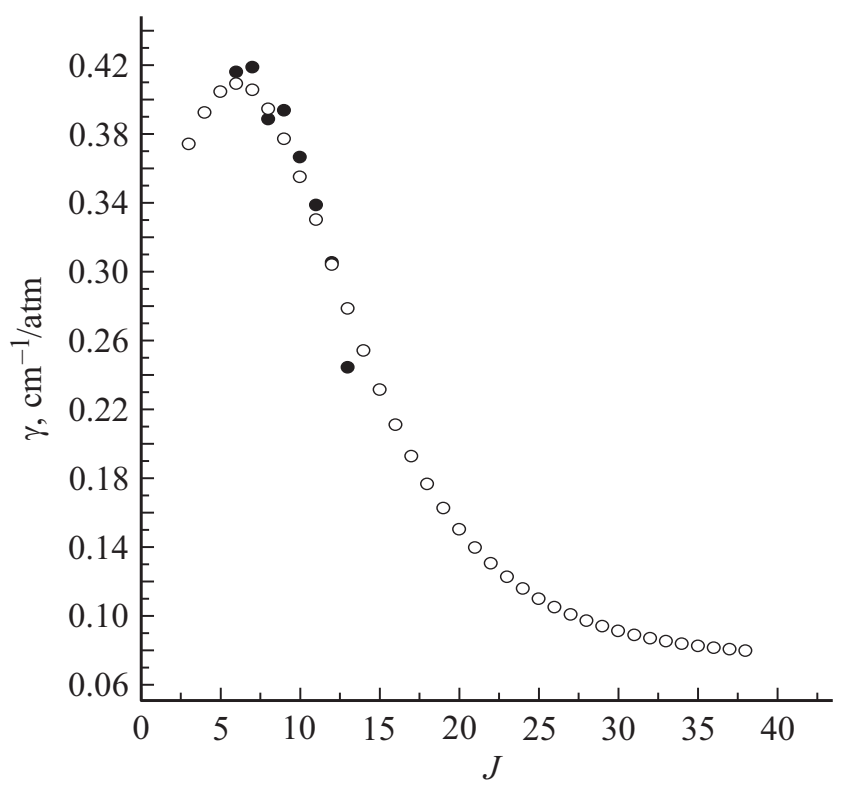

Рис. 2. Экспериментальные [12] (темные символы) и вычисленные (светлые символы) коэффициенты самоуширения линий $[J 3 J-3] \rightarrow[J+14 J-2]$ полосы $v_{2}$ молекулы водяного пара.

и экспериментальные [12] значения $\gamma$ для двух колебательных полос, $2 v_{2}$ и $3 v_{2}$, молекулы $\mathrm{H}_{2} \mathrm{O}$. Данные для этих полос не использовались для определения параметров модели $\gamma$ (sur).

Согласие с экспериментальными данными вполне хоpoшеe.
Согласно результатам (10), (11) статистического анализа для ряда линий $\chi_{\gamma}>50 \%$, т.е. наблюдается большое отклонение значений $\gamma$, вычисленных с помощью модели $\gamma$ (sur), от экспериментальных (или вычисленных по полуклассическому методу). Этот факт не обязательно является следствием неадекватности модели, но может быть связан и с наличием типографских

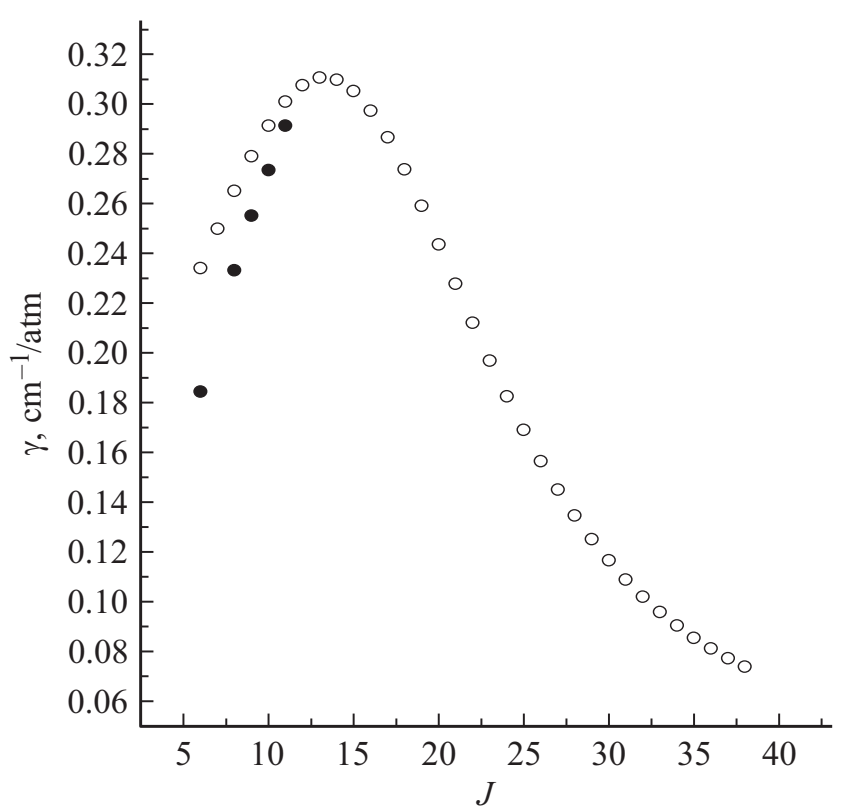

Рис. 3. Экспериментальные [12] (темные символы) и вычисленные (светлые символы) коэффициенты самоуширения линий $[J 6 J-5] \rightarrow[J+17 J-6]$ полосы $v_{2}$ молекулы водяного пара.

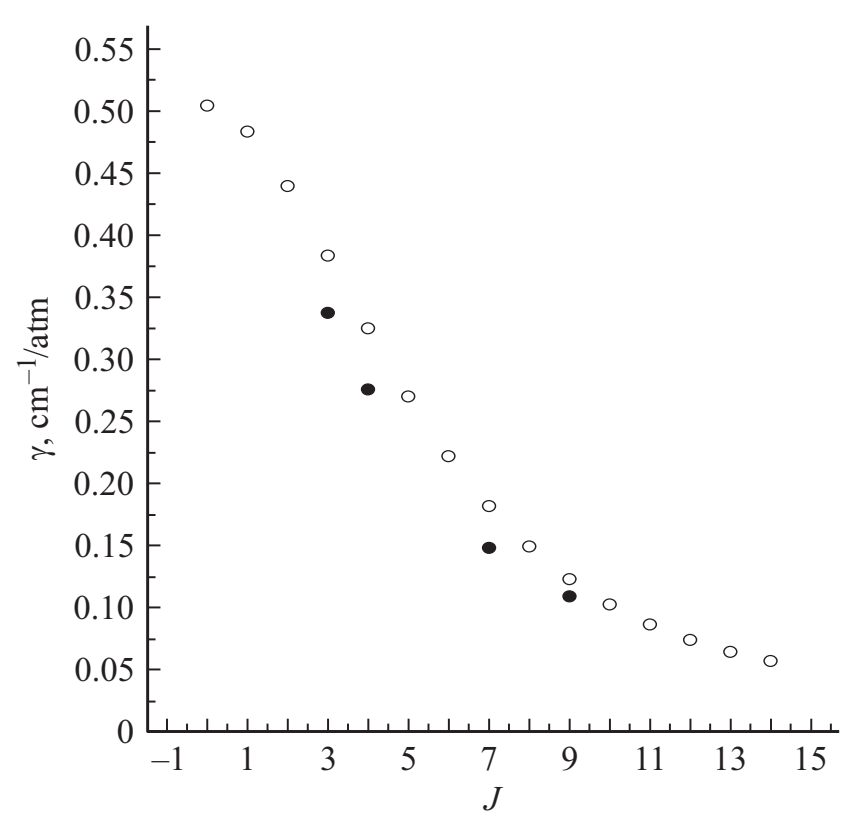

Рис. 4. Экспериментальные [12] (темные символы) и вычисленные (светлые символы) коэффициенты самоуширения линий $\left[J K_{a}=J K_{c}=0\right] \rightarrow\left[J+1 K_{a}=J+1 K_{c}=1\right]$ полосы $v_{2}$ молекулы водяного пара. 


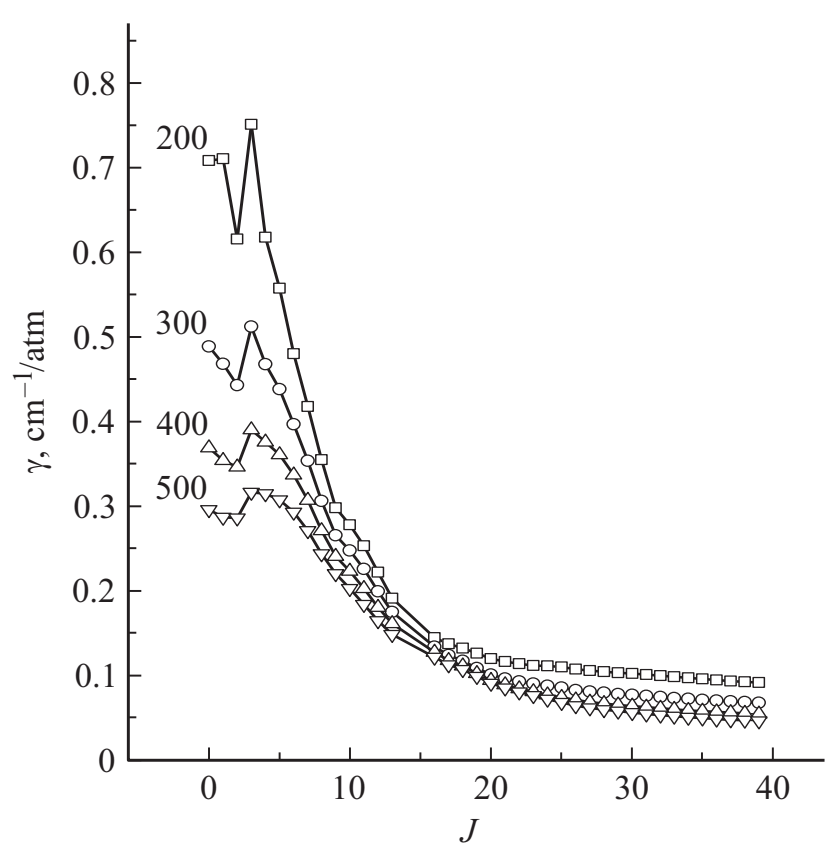

Pис. 5. Асимптотическое поведение коэффициентов самоуширения $\gamma$ линий для вращательной ветви $[J 0 J] \rightarrow[J+11 J+1]$ полосы $v_{2}$ молекулы $\mathrm{H}_{2} \mathrm{O}$, вычисленных полуклассическим методом [7,8] для температур $T=200,300,400,500 \mathrm{~K}$.

Таблица 5. Сравнение вычисленных по модели (7)-(9) и экспериментальных [12] значений $\gamma\left(\mathrm{cm}^{-1} / \mathrm{atm}\right)$ для полос $2 v_{2}$, $3 v_{2}$ - молекулы $\mathrm{H}_{2} \mathrm{O}(T=296 \mathrm{~K})$

\begin{tabular}{|c|c|c|c|c|}
\hline$J_{f} K_{a f} K_{c f}$ & $J_{i} K_{a i} K_{c i}$ & $\gamma(\exp )$ & $\gamma(\text { sur })^{1}$ & $\gamma(\text { sur })^{2}$ \\
\hline $2 v_{2}$ & & & & \\
\hline 524 & 615 & 0.399 & 0.435 & 0.420 \\
\hline 321 & 330 & 0.399 & 0.406 & 0.424 \\
\hline 835 & 826 & 0.429 & 0.434 & 0.403 \\
\hline 624 & 515 & 0.454 & 0.435 & 0.419 \\
\hline $\begin{array}{l}\chi_{\mathrm{av}} \\
3 v_{2}\end{array}$ & & & $4.0 \%$ & $6.3 \%$ \\
\hline 818 & 909 & 0.284 & 0.302 & 0.282 \\
\hline 716 & 827 & 0.353 & 0.357 & 0.356 \\
\hline 606 & 717 & 0.352 & 0.355 & 0.339 \\
\hline 827 & 918 & 0.267 & 0.343 & 0.328 \\
\hline 616 & 707 & 0.362 & 0.366 & 0.339 \\
\hline 404 & 515 & 0.425 & 0.413 & 0.399 \\
\hline 625 & 716 & 0.358 & 0.402 & 0.383 \\
\hline 414 & 505 & 0.420 & 0.420 & 0.399 \\
\hline 303 & 414 & 0.465 & 0.437 & 0.426 \\
\hline 202 & 313 & 0.428 & 0.454 & 0.450 \\
\hline 101 & 212 & 0.477 & 0.466 & 0.467 \\
\hline 212 & 303 & 0.451 & 0.452 & 0.450 \\
\hline 101 & 110 & 0.435 & 0.471 & 0.479 \\
\hline 221 & 110 & 0.450 & 0.456 & 0.456 \\
\hline$\chi_{\mathrm{av}}$ & & & $5.5 \%$ & $5.7 \%$ \\
\hline
\end{tabular}

Примечание. ${ }^{1,2}$ - вычисления с параметрами из табл. 3 и 4 соответственно. или иных ошибок в литературных данных для экспериментальных значений коэффициентов $\gamma$. Большие отклонения (c $\chi>50 \%)$ коэффициентов самоуширения, вычисленных с помощью модели $\gamma$ (sur) и параметров из табл. 4, от экспериментальных значений есть только для 4 линий, показанных в табл. 6.

Для последних двух линий из этой таблицы возможен эффект сужения линий, который в работе не учитывается, экспериментальные значения $\gamma$ для первых двух линий, по-видимому, ошибочны.

\section{Заключение}

В работе вычислены коэффициенты самоуширения $\gamma$ линий водяного пара для переходов на высоковозбужденные вращательные состояния с $J_{i} \leq 41, K_{a i} \leq 25$ двенадцати колебательных полос из спектрального диапазона $0.7-14000.0 \mathrm{~cm}^{-1}$. Вычисления проведены в рамках полуклассического метода [7,8]. Для представления вычисленных коэффициентов самоуширения с $J_{i} \leq 41$, $K_{a i} \leq 13$ и их экспериментальных значений предложено использовать аналитическую модель (7)-(9). Параметры модели получены в двух вариантах подгонки выражений (7)-(9) к вычисленным и экспериментальным коэффициентам $\gamma$. В первом варианте параметры определены для отдельных вращательных ветвей $\left[J_{i} K_{a i} K_{c i}\right] \rightarrow\left[J_{f} K_{a f} K_{c f}\right]$ с фиксированным значением квантового числа $K_{a i}=0, \ldots, 10$ всех колебательных полос. Эти параметры приведены в табл. 3. Они позволяют проводить расчеты $\gamma$ в таких ветвях для произвольных $J_{i} \leq 41$ с фиксированным значением $K_{a i}$. Во втором варианте параметры модели (7)-(9) определены одновременно для всех ветвей. Такие параметры приведены в табл. 4. Они позволяют проводить экстраполяцию значений $\gamma$ на переходы с различными $K_{a i} \leq 15$.

Таблица 3 предпочтительно использовать при расчетах $\gamma$ для отдельных ветвей с $K_{a i} \leq 10$, табл. $4-$ для расчетов $\gamma$ с $K_{a i}>10$.

Параметры модели (7)-(9) из табл. 3 и 4 содержат информацию о 34195 и 34635 коэффициентах $\gamma$ соответственно. Параметр $t_{22}$ из таблиц позволяет проводить экстраполяцию $\gamma$ на колебательные полосы с различным значением квантового числа $v_{2}$ конечного колебательного состояния. Значения параметров $t_{21}=t_{23}=0$ из этих таблиц указывает на то, что коэффициенты $\gamma$ слабо зависят от квантовых чисел $v_{1}$ и $v_{3}$, связанных с валентными колебаниями.

Согласно [2], для различных приложений полуширины линий должны быть известны с погрешностью, меньшей 5\%. Оценка точности экспериментальных данных для полуширин линий $\mathrm{H}_{2} \mathrm{O}$, проведенная в [2], показывает, что эти данные не удовлетворяют требуемой точности. Более того, в случае самоуширения линий водяного пара в [2] получена оценка экспериментальной погрешности от 20 до 25\%. Погрешность расчета коэффициентов $\gamma$ по модели (7)-(9), определяемая величиной $\chi_{\mathrm{av}}$, меняется от 6.2 до $10.5 \%$ для отдельных 
Таблица 6. Линии с большим отклонение $(\chi>50 \%)$ значений $\gamma\left(\right.$ в $\left.\mathrm{cm}^{-1} / \mathrm{atm}\right)$, вычисленных с помощью модели (7)-(9), от экспериментальных $[12,14]$

\begin{tabular}{l|c|c|c|c|c|c}
\hline Полоса & $J_{f} K_{a f} K_{c f}$ & $J_{i} K_{a i} K_{c i}$ & {$[12,14]$} & $\gamma($ sur $)$ & $\chi, \%$ & $\gamma_{\text {calc }}^{1}$ \\
\hline$\nu_{2}+\nu_{3}$ & 404 & 515 & 0.2660 & 0.4222 & 58.72 & 0.4877 \\
$\nu_{2}$ & 836 & 909 & 0.2110 & 0.3418 & 61.99 & 0.3438 \\
$\nu_{2}$ & 132121513 & 0.1468 & 0.2209 & 50.47 & 0.1794 \\
$\nu_{2}$ & 1615 & 15214 & 0.1128 & 0.1846 & 63.65 & 0.1553
\end{tabular}

Примечание. ${ }^{1}-$ вычисление по полуклассическому методу, $T=296 \mathrm{~K}$.

вращательных ветвей, $\chi_{\mathrm{av}}=9.3 \%$ для всех ветвей одновременно.

Модель (7)-(9) не связана с межмолекулярным потенциалом взаимодействия, волновыми функциями и частотами переходов в молекуле $\mathrm{H}_{2} \mathrm{O}$, поэтому позволяет проводить расчеты для переходов более высокого спектрального диапазона, связанного с возбуждением изгибной моды колебаний (зависимость $\gamma$ от валентных колебаний не обнаружена). Примеры расчета асимптотического поведения вычисленных в модели (7)-(9) коэффициентов самоуширения представлены на рис. 1-5, пример расчета $\gamma$ для линий, не включенных в анализ, показан в табл. 5. Кроме этого, модель (7)-(9) может быть использована для расчета зависимости коэффициентов $\gamma$ от температуры (в интервале $200 \leq T \leq 500 \mathrm{~K}$ ).

\section{Список литературы}

[1] Claveau C., Henry A., Hurtmans D.,Valentin A. // JQSRT. 2001. V. 68. P. 273.

[2] Gamache R.R., Hartmann J.-M., Rosenmann L. // JQSRT. 1994. V. 52. P. 481.

[3] Стариков В.И., Лаврентьева Н.Н. Столкновительное уширение спектральных линий поглощения молекул атмосферных газов. Томск: Изд. ИОА СО РАН, 2006. 307 с.

[4] Buldyreva J., Lavrent'eva N.N., Starikov V.I. Collisional Line Broadening and Shifting of Atmosphyric Gase. A Practical Guide for Line Shape Modeling by Current Semi-classical Approaches. London: Imperial College Press, 2010. 292 p.

[5] Antony B.K., Neshyba S., Gamache R.R. // JQSRT. 2007. V. 105. P. 148.

[6] Протасевич А.Е., Михайленко С.Н., Стариков В.И. // Оптика атмосферы и океана. 2002. Т. 15. С. 790.

[7] Robert D., Bonamy. // J. Phys. (Paris) 1979. V. 40. P. 923.

[8] Leavitt R.P // J. Chem. Phys. 1980. V. 73. N 11. P. 5432.

[9] Mengel M., Jensen P. // J.Mol.Spectrosc. 1995. V. 169 P. 73.

[10] Starikov V.I // J. Mol. Spectrosc. 2001. V. 206. P. 166.

[11] Starikov V.I., Petrova T.M., Solodov A.M., Solodov A.A., Deichuli V.M. // Spectrochim. Acta A. 2019. V. 210. P. 275.

[12] Toth R.A., Brown L.R., Plymate C. // JQSRT. 1998. V. 59. P. 529.

[13] Devi V.M., Gamache R.R., Vispoel B., Renaud C.L. Bener D.C., Smith M.A.H., Blake T.A., Sams R.L. // J. Mol. Spectrosc. 2018. V. 348. P. 13-36.

[14] Toth R.A. // JQSRT. 2005. V. 94. P. 1.

[15] Grossmann B.E., Browell E.V. // J. Mol. Spectr. 1989. V. 136. P. 264-294.
[16] Стариков В.И., Протасевич А.Е. // Опт. и спектр. 2005. T. 38 . C. 368. 